\title{
Uniqueness and Nonuniqueness in the Einstein Constraints
}

\author{
Harald P. Pfeiffer ${ }^{1}$ and James W. York, Jr. ${ }^{2}$ \\ ${ }^{1}$ Theoretical Astrophysics 130-33, California Institute of Technology, Pasadena, California 91125, USA \\ ${ }^{2}$ Department of Physics, Cornell University, Ithaca, New York, 14853, USA
}

(Received 28 April 2005; published 26 August 2005)

\begin{abstract}
The conformal thin-sandwich (CTS) equations are a set of four of the Einstein equations, which generalize the Laplace-Poisson equation of Newton's theory. We examine numerically solutions of the CTS equations describing perturbed Minkowski space, and find only one solution. However, we find two distinct solutions, one even containing a black hole, when the lapse is determined by a fifth elliptic equation through specification of the mean curvature. While the relationship of the two systems and their solutions is a fundamental property of general relativity, this fairly simple example of an elliptic system with nonunique solutions is also of broader interest.
\end{abstract}

DOI: 10.1103/PhysRevLett.95.091101

PACS numbers: 04.20.Ex, 04.20.Cv, 04.25.Dm

Within a space-plus-time decomposition [1,2], Einstein's equations, just as Maxwell's equations, split into evolution equations and initial value equations. The latter constrain the initial data and are generally solved by the use of potentials that satisfy elliptic equations. These potentials are expected to be unique, given suitable boundary conditions. For the Einstein constraints, indeed, in all cases in which existence of solutions has been proved, the solution was also shown to be unique.

However, we demonstrate in this Letter that an important method of solving the Einstein constraints through potentials, the extended conformal thin-sandwich (XCTS) equations [3,4], admits nonunique solutions. Two solutions appear to exist even for arbitrarily small perturbations away from Minkowski space; one solution is just perturbed Minkowski space, but the second one even contains a black hole for sufficiently small perturbation. The XCTS system thus provides a fairly simple example of a nonlinear elliptic system with nonunique solutions, which may be of interest outside general relativity. Because the XCTS system lies at the heart of most modern schemes to construct binary neutron star [5-8] and binary black hole [9-12] initial data, our result is also relevant to the astrophysical problem of computing inspiral waveforms for compact objects.

In general relativity, the initial data are the induced Riemannian metric $g_{i j}$ and the extrinsic curvature $K_{i j}$ on a spacelike hypersurface. The data $\left(g_{i j}, K_{i j}\right)$ must satisfy four nonlinear constraint equations, and there are now two equivalent, complete approaches with which we can solve the constraints. One methods seeks $\left(g_{i j}, K_{i j}\right)$ directly [4], while the other, the "conformal thin-sandwich" (CTS) method [3], seeks to construct $\left(g_{i j}, \partial_{t} g_{i j}\right)$. In general relativity, this difference is not trivial: $K_{i j}$ depends only on the embedding of the slice (see, e.g., [13]) while $\partial_{t} g_{i j}$ depends also on the ambient coordinate neighborhood of the slice. Both methods result in four coupled elliptic equations, and many existence and uniqueness results are known [14].

The extended conformal thin-sandwich formalism adds a fifth elliptic equation for the lapse function, which arises from specification of the time derivative of the mean curvature $K \equiv K_{i j} g^{i j}$. In the extended system, the free data consists entirely of "variable and velocity" pairs $(q, \dot{q})$ [4], as desirable for a Lagrangian viewpoint, or a thin-sandwich viewpoint. However, the fifth equation couples strongly all five equations of the extended system, complicating mathematical analysis, so that to our knowledge, there are no rigorous mathematical results for the extended system, neither for existence nor for uniqueness. Such difficulties, as well as the nonuniqueness results reported in this Letter are not present for the standard initial value problem, but arise when going beyond the mere initial value problem by placing demands on the slicing in the ambient spacetime via specification of $\partial_{t} K$. Similar issues connected with general relativity have been called traditionally the "problem of time."

The CTS equations can be written as

$$
\begin{array}{r}
\tilde{\nabla}^{2} \psi-\frac{1}{8} \tilde{R} \psi-\frac{1}{12} K^{2} \psi^{5}+\frac{1}{8} \psi^{-7} \tilde{A}^{i j} \tilde{A}_{i j}=0, \\
\tilde{\nabla}_{j}\left(\frac{1}{2 \tilde{N}}(\tilde{\mathbb{L}} \beta)^{i j}\right)-\frac{2}{3} \psi^{6} \tilde{\nabla}^{i} K-\tilde{\nabla}_{j}\left(\frac{1}{2 \tilde{N}} \tilde{u}^{i j}\right)=0 .
\end{array}
$$

Here, $\tilde{\nabla}$ and $\tilde{R}$ are the covariant derivative and the trace of the Ricci tensor associated with the conformal metric $\tilde{g}_{i j}$, which is related to the physical metric by $g_{i j}=\psi^{4} \tilde{g}_{i j}$. Furthermore,

$$
\tilde{A}^{i j} \equiv \frac{1}{2 \tilde{N}}\left[(\tilde{\mathbb{L}} \beta)^{i j}-\tilde{u}^{i j}\right]=\psi^{10}\left(K^{i j}-\frac{1}{3} g^{i j} K\right)
$$

represents the conformal trace-free extrinsic curvature, $(\tilde{\mathbb{L}} \beta)^{i j} \equiv 2 \tilde{\nabla}^{(i} \beta^{j)}-2 / 3 \tilde{g}^{i j} \tilde{\nabla}_{k} \beta^{k}$ is the conformal longitudinal operator, and the traceless tensor $\tilde{u}_{i j} \equiv \partial_{t} \tilde{g}_{i j}$ represents the time derivative of the conformal metric. Once the free data $\left(\tilde{g}_{i j}, \tilde{u}_{i j} ; K, \tilde{N}\right)$ are chosen, Eqs. (1) and (2) are elliptic and determine the conformal factor $\psi$ and the shift vector $\beta^{i}$. The conformal lapse $\tilde{N}$ is related to the physical lapse by $N=\psi^{6} \tilde{N}$. 
The elliptic equation for the lapse, which follows from the Einstein evolution equation for $K$, can be written as

$$
\tilde{\nabla}^{2}\left(\tilde{N} \psi^{7}\right)-\left(\tilde{N} \psi^{7}\right)\left(\frac{1}{8} \tilde{R}+\frac{5}{12} K^{2} \psi^{4}+\frac{7}{8} \psi^{-8} \tilde{A}^{i j} \tilde{A}_{i j}\right)=-\psi^{5}\left(\partial_{t} K-\beta^{k} \partial_{k} K\right)
$$

Viewing $\partial_{t} K$ instead of $\tilde{N}$ as freely specifiable, the free data become $\left(\tilde{g}_{i j}, \tilde{u}_{i j} ; K, \partial_{t} K\right)$, and one must solve the coupled elliptic system Eqs. (1), (2), and (4). This is the XCTS system.

We will show nonuniqueness by explicitly constructing two solutions for a certain choice of free data. This choice is based on a linearized quadrupolar gravitational wave [15] and follows very closely [16]. We set

$$
\begin{array}{rlrl}
\tilde{g}_{i j} & =f_{i j}+\left.\tilde{\mathcal{A}} h_{i j}\right|_{t=0}, \\
\tilde{u}_{i j} & =\left.\tilde{\mathcal{A}} \mathrm{TF}_{\tilde{g}} \partial_{t} h_{i j}\right|_{t=0}, \\
K & =0, & \\
\tilde{N} & =1, \quad \text { for CTS, } \\
\partial_{t} K & =0, \quad \text { for XCTS, }
\end{array}
$$

where $f_{i j}$ represents the Euclidean metric, $\mathrm{TF}_{\tilde{g}}$ means the trace-free part with respect to $\tilde{g}_{i j}$, and,

$h_{i j} \mathrm{~d} x^{i} \mathrm{~d} x^{j}=-8 r K \cos \Theta \sin ^{2} \Theta d r d \phi-2 r^{2} L \sin ^{3} \Theta d \phi d \Theta$.

The functions $K$ and $L$ are given by

$$
\begin{aligned}
& K=r^{-2} F^{(2)}-3 r^{-3} F^{(1)}+3 r^{-4} F, \\
& L=-r^{-1} F^{(3)}+2 r^{-2} F^{(2)}-3 r^{-3} F^{(1)}+3 r^{-4} F,
\end{aligned}
$$

where $F=F(r+t)=e^{-\left(r+t-r_{0}\right)^{2} / w^{2}}$ denotes the radial profile, and $F^{(n)} \equiv d^{n} F(x) / d x^{n}$. For small $\tilde{\mathcal{A}}$, this choice of $h_{i j}$ corresponds to a localized incoming gravitational wave at radius $r_{0} \equiv 20$ with width $w \equiv 1$, having odd parity and azimuthal quantum number $M=0$.

Being based on a solution to the linearized Einstein equations, the free data (5) satisfy the conformal thinsandwich equations (1), (2), and (4), to linear order in $\tilde{\mathcal{A}}$, but violate them to $\mathcal{O}\left(\tilde{\mathcal{A}}^{2}\right)$. Consequently, the XCTS equations have a solution which corrects the free data by $\mathcal{O}\left(\tilde{\mathcal{A}}^{2}\right)$, as demonstrated in [16]. However, this is not the full story.

We employ the spectral elliptic solver described in [17] to solve Eqs. (1), (2), and (4), in a computational domain with outer radius $10^{8}$ with Dirichlet boundary conditions, $\psi=N=1, \beta^{i}=0$. For smooth problems, like the ones considered here, such a spectral method results in exponential convergence, and allows construction of highly accurate solutions. All solutions presented in this Letter converge indeed exponentially; generally, the maximum residual of both Hamiltonian and momentum constraint is $\$ 10^{-6}$, and other quantities we mention below have converged to a similar level. The convergence rate diminishes along the "upper branch" of the extended system at amplitudes $\tilde{\mathcal{A}} \lesssim 0.2$, but the relative errors remain $\lesssim 10^{-4}$ even at $\tilde{\mathcal{A}}=0.02$. In all figures, numerical errors are much smaller than the line thickness, so that essentially the "true" analytical solutions are plotted.

Figure 1 presents solutions to the standard conformal thin-sandwich equations for various amplitudes $\tilde{\mathcal{A}}$. The conformal factor $\psi$ diverges as $\tilde{\mathcal{A}}$ approaches some critical amplitude. For our particular choice $\tilde{N}=1, K=0$, Eqs. (1) and (2) reduce to a set of equations which is very well understood mathematically [18-20]: Solutions are unique and exist iff the Yamabe constant of $\tilde{g}_{i j}$ is positive. Figure 1 suggests that the Yamabe constant of the metric Eq. (5a) is positive for small $\tilde{\mathcal{A}}$ and changes sign at $\tilde{\mathcal{A}}=$ $\tilde{\mathcal{A}}_{c, 4}$, similar to an example given in [19].

The situation is very different when solving the extended system. Figure 2 presents the plot analogous to Fig. 1 for this case. As in the standard system, we have not been able to find solutions beyond some critical amplitude. However, here the similarity ends. For the extended system, the critical amplitude $\tilde{\mathcal{A}}_{c, 5} \approx 0.30422$ is much smaller than for the standard system. Therefore, existence of solutions cannot depend simply on the sign of the Yamabe constant of $\tilde{g}_{i j}$. Furthermore, as $\delta \tilde{\mathcal{A}} \equiv \tilde{\mathcal{A}}_{c, 5}-\tilde{\mathcal{A}}$ becomes small, $\max \psi$ varies as $(\delta \tilde{\mathcal{A}})^{1 / 2}$ and approaches a finite limit $\psi_{\text {crit }} \approx 1.0999$ (the numerical values for $\tilde{\mathcal{A}}_{c, 5}$ and $\psi_{\text {crit }}$ were computed by fitting a parabola). The log-log plot in the inset of Fig. 2 confirms these statements. Inspection of the solutions shows that as $\delta \tilde{\mathcal{A}} \rightarrow 0$ all variables vary in proportion to $(\delta \tilde{\mathcal{A}})^{1 / 2}$ at any spatial coordinate location $x$,

$$
u_{-}(\tilde{\mathcal{A}} ; x) \approx u_{\text {crit }}(x)-v_{\text {crit }}(x) \sqrt{\delta \tilde{\mathcal{A}}}
$$

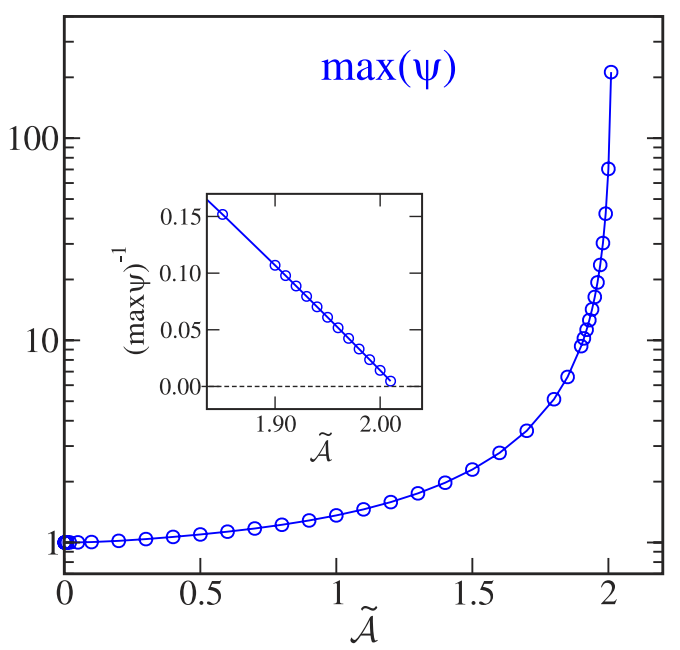

FIG. 1 (color online). Solutions of the standard (four) conformal thin-sandwich equations vs the conformal amplitude. As the critical amplitude $\mathcal{A}_{c, 4} \approx 2.01$ is approached, the conformal factor grows to infinity. 


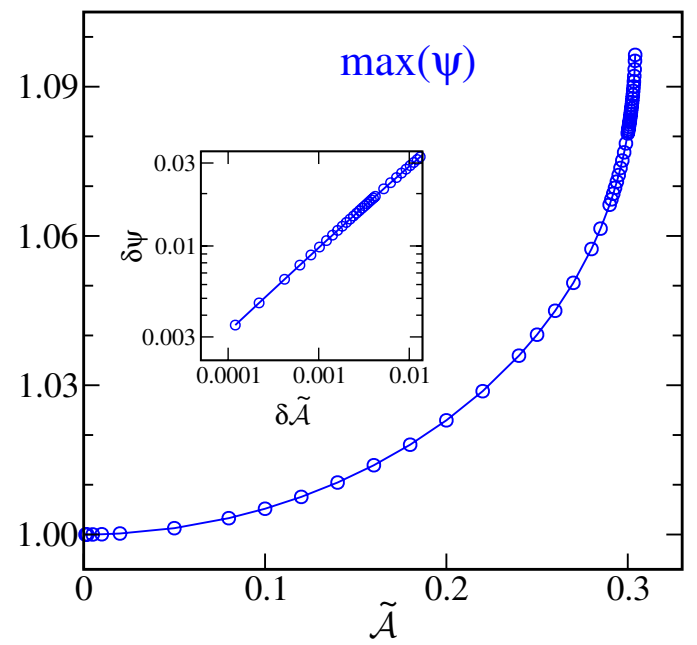

FIG. 2 (color online). Solutions of the extended (five) conformal thin-sandwich equations vs conformal amplitude. The conformal factor remains finite as the critical amplitude $\tilde{\mathcal{A}}_{c, 5} \approx 0.304$ is approached, as confirmed by the inset $[\delta \tilde{\mathcal{A}}=$ $\left.\tilde{\mathcal{A}}_{c, 5}-\tilde{\mathcal{A}}, \delta \psi=\psi_{\text {crit }}-\max (\psi)\right]$.

Here, $u_{-}, u_{\text {crit }}$, and $v_{\text {crit }}$ represent the vector of all five variables $\left(\psi, \beta^{i}, \tilde{N}\right)$, and the notation indicates that $u_{-}$ depends on both $\tilde{\mathcal{A}}$ and the spatial coordinates $x$, whereas $u_{\text {crit }}$ and $v_{\text {crit }}$ are independent of $\tilde{\mathcal{A}}$ sufficiently close to $\tilde{\mathcal{A}}_{c, 5}$.

Because of the parabolic behavior close to $\tilde{\mathcal{A}}_{c, 5}$, we expect a second branch of solutions, generalizing Eq. (6) to

$$
u_{ \pm}(\tilde{\mathcal{A}} ; x) \approx u_{\text {crit }}(x) \pm v_{\text {crit }}(x) \sqrt{\delta \tilde{\mathcal{A}}}
$$

The solutions found so far in Fig. 2 were obtained with trivial initial guess $\psi=N=1, \beta^{i}=0$, and constitute the lower branch $u_{-}$only; to converge to $u_{+}$, an initial guess sufficiently close to $u_{+}$is necessary. Differentiation of Eq. (6) with respect to $\tilde{\mathcal{A}}$ yields $v_{\text {crit }}=$ $2(\delta \tilde{\mathcal{A}})^{1 / 2} d u_{-} / d \tilde{\mathcal{A}}$, so that, sufficiently close to $\tilde{\mathcal{A}}_{c, 5}$,

$$
u_{+}(\tilde{\mathcal{A}} ; x) \approx u_{-}(\tilde{\mathcal{A}} ; x)+4 \delta \tilde{\mathcal{A}} \frac{d u_{-}(\tilde{\mathcal{A}} ; x)}{d \tilde{\mathcal{A}}} .
$$

Approximating $d u_{-} / d \tilde{\mathcal{A}}$ by a finite-difference quotient of solutions with $\tilde{\mathcal{A}}=0.300$ and $\tilde{\mathcal{A}}=0.301$, we compute the right-hand side of Eq. (8) and use it as an initial guess for the elliptic solver at $\tilde{\mathcal{A}}=0.300$. The solver now converges to a different solution; for example, $\max \psi \approx$ 1.12. This solution $u_{+}$for one $\tilde{\mathcal{A}}$ is then used as an initial guess for solutions with neighboring $\tilde{\mathcal{A}}$ and repeating this process, we move along the upper branch and compute solutions $u_{+}$for a wide range of $\tilde{\mathcal{A}}$. Eventually, Fig. 3 emerges, which shows clearly the two distinct branches merging in a parabola at $\tilde{\mathcal{A}}_{c, 5}$.

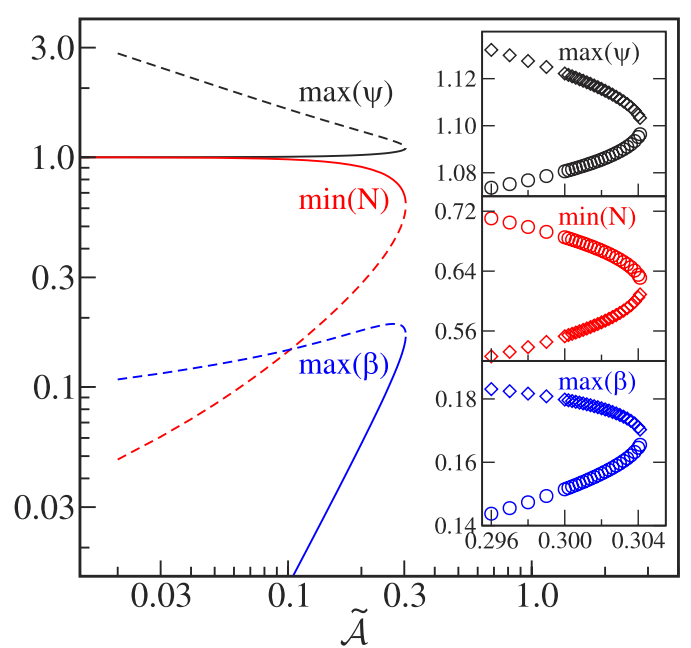

FIG. 3 (color online). Maximum of the conformal factor, minimum of the physical lapse $N=\psi^{6} \tilde{N}$, and maximal magnitude of the shift vs conformal (unphysical) amplitude. Solid/ dashed curves represent the lower/upper branch. The insets show enlargements around the critical point; circles/diamonds denote individual solutions along the lower/upper branch.

The solutions $u_{+}$are quite remarkable. They exist over a wide range of $\tilde{\mathcal{A}}$, down to small $\tilde{\mathcal{A}}$. As $\tilde{\mathcal{A}}$ decreases, $\psi$ increases everywhere; the increase is particularly pronounced close to the maximum of $\psi$ which occurs in a ring in the equatorial plane at a radius close to $r_{0}$. Consequently, with decreasing $\tilde{\mathcal{A}}$, the maxima of $\psi$ become increasingly pronounced and concentrated around $r \approx r_{0}$ in the equatorial plane. This necessitates higher angular resolution in latitude and diminishes the convergence rate of the spectral expansion, so that we choose to stop at $\tilde{\mathcal{A}}=0.02$. We note, however, that we do not see any fundamental indication that the branch $u_{+}$terminates; it appears that $u_{+}$extends to arbitrarily small $\tilde{\mathcal{A}}$, with ever increasing $\psi$. The physical (Arnowitt-Deser-Misner [1]) energy $E$ of the computed initial data sets varies approximately proportionally to $\max \psi-1$ along $u_{+}$(see also Fig. 4). At $\tilde{\mathcal{A}}=0.02, E=50.012$ (in units in which $r_{0}=$ 20). This data set also contains an apparent horizon with mass $M=\sqrt{\text { Area } / 16 \pi}=49.872$. The apparent horizon is oblate with equatorial radius 28.8 and polar radius 21.1. Apparent horizons are present for $\tilde{\mathcal{A}} \lesssim 0.025$.

Figure 3 shows clearly that the extended system admits two solutions for the same free (conformal) data. However, the physical properties of the initial data sets depend on the physical quantities $\left(g_{i j}, K_{i j}\right)$; for example, the metric takes the form

$$
g_{i j}=\psi^{4} f_{i j}+\left(\tilde{\mathcal{A}} \psi^{4}\right) h_{i j}
$$

This can be interpreted as the superposition of a gravitational wave $h_{i j}$ with physical amplitude $\tilde{\mathcal{A}} \psi^{4}$ on a physical background $\psi^{4} f_{i j}$. The conformal factor $\psi$ is a function of 


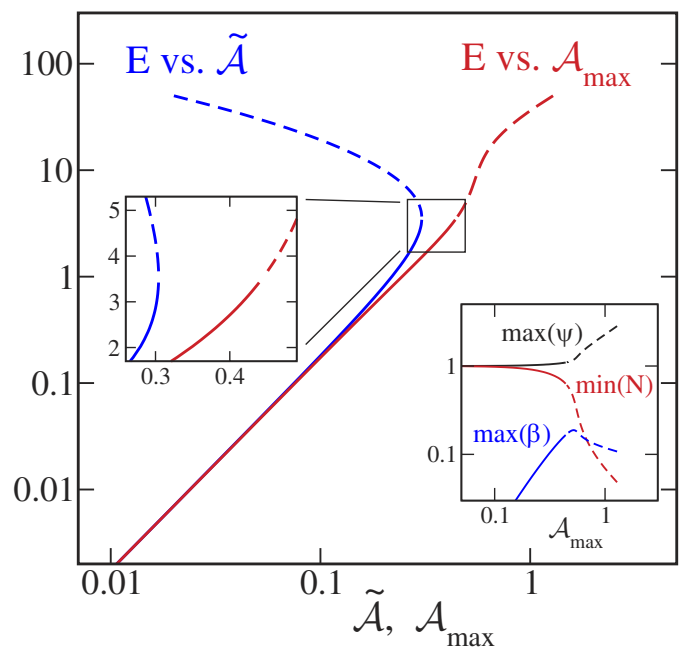

FIG. 4 (color online). Energy $E$ vs unphysical amplitude $\tilde{\mathcal{A}}$ and physical amplitude $\mathcal{A}_{\text {max }}$. The left inset shows an enlargement around the critical point. The right inset plots the data of Fig. 3 vs $\mathcal{A}_{\max }$.

space, and therefore $\tilde{\mathcal{A}} \psi^{4}$ is also spatially dependent. To simplify, we take the maximum of $\psi$, which coincides with the location of the gravitational wave at radius $r_{0}=20$, and define a representative physical amplitude by $\mathcal{A}_{\max } \equiv$ $\tilde{\mathcal{A}} \max \psi^{4}$.

Figure 4 plots energy $E$ both vs $\tilde{\mathcal{A}}$ and $\mathcal{A}_{\text {max }}$; the qualitative difference between both definitions of amplitude is apparent: The unphysical amplitude changes "direction" along the sequence of solutions leading to the largest energy at smallest $\tilde{\mathcal{A}}$. In contrast, $\mathcal{A}_{\max }$ is continuously increasing, precisely one solution corresponds to each value of $\mathcal{A}_{\max }$, and large energies are obtained at large $\mathcal{A}_{\max }$. The family of solutions passes smoothly through the critical point, and even for arbitrarily large $\mathcal{A}_{\text {max }}$, a solution seems to exist. Thus, $\mathcal{A}_{\max }$ represents the amplitude of the physical perturbation much more faithfully than $\tilde{\mathcal{A}}$.

We see that uniqueness of the problem is not a straightforward issue. It depends on the question: for a given set of free data (this is, for a given value of $\tilde{\mathcal{A}}$ ), the elliptic equations may have two solutions $\left(\tilde{\mathcal{A}}<\tilde{\mathcal{A}}_{c, 5}\right.$ ) or no solution at all $\left(\tilde{\mathcal{A}}>\tilde{\mathcal{A}}_{c, 5}\right)$. However, for each value of the physical amplitude $\mathcal{A}_{\text {max }}$ as described, precisely one solution exists within the family of solutions considered here.

While the relationship between uniqueness and nonuniqueness of the XCTS system as discussed above is intriguing, the major result of this work lies in the fact that the XCTS system may have two solutions for the same free data. Besides the obvious importance of this result to workers in mathematical relativity, we note that this issue may complicate construction of astrophysical compact object initial data, which relies on some form of the XCTS equations [5-12]. Furthermore, the most common elliptic gauge conditions [21] include precisely Eq. (4) to determine the lapse function.

The authors thank Lee Lindblom, Lawrence Kidder, and Mark Scheel for helpful discussions; the numerical code has been developed in collaboration with Lawrence Kidder and Mark Scheel. This work was supported in part by NSF Grant Nos. PHY-0244906 and PHY-0099568 to Caltech and Grant Nos. PHY-0407762, PHY-0311817, and PHY0216986 to Cornell. H. P. gratefully acknowledges support from the Sherman Fairchild Foundation.

[1] R. Arnowitt, S. Deser, and C. W. Misner, in Gravitation, edited by L. Witten (Wiley, New York, 1962).

[2] J.W. York, Jr., in Sources of Gravitational Radiation, edited by L.L. Smarr (Cambridge University Press, Cambridge, England, 1979), p. 83.

[3] J. W. York, Jr., Phys. Rev. Lett. 82, 1350 (1999).

[4] H. P. Pfeiffer and J. W. York, Jr., Phys. Rev. D 67, 044022 (2003).

[5] J. R. Wilson and G. J. Mathews, in Frontiers in Numerical Relativity, edited by C. R. Evans, L. S. Finn, and D.W. Hobill (Cambridge University Press, Cambridge, England, 1989), p. 306.

[6] G. B. Cook, S. L. Shapiro, and S.A. Teukolsky, Phys. Rev. D 53, 5533 (1996).

[7] T. W. Baumgarte, G. B. Cook, M. A. Scheel, S. L. Shapiro, and S. A. Teukolsky, Phys. Rev. Lett. 79, 1182 (1997).

[8] P. Marronetti, G. J. Mathews, and J. R. Wilson, Phys. Rev. D 58, 107503 (1998).

[9] E. Gourgoulhon, P. Grandclément, and S. Bonazzola, Phys. Rev. D 65, 044020 (2002).

[10] P. Grandclément, E. Gourgoulhon, and S. Bonazzola, Phys. Rev. D 65, 044021 (2002).

[11] G. B. Cook, Phys. Rev. D 65, 084003 (2002).

[12] G. B. Cook and H. P. Pfeiffer, Phys. Rev. D 70, 104016 (2004).

[13] Y. Choquet-Bruhat and J. W. York, in General Relativity and Gravitation: An Einstein Centenary Survey, edited by A. Held (Plenum, New York, 1980), Vol. 1, p. 99.

[14] R. Bartnik and J. Isenberg, gr-qc/0405092.

[15] S. A. Teukolsky, Phys. Rev. D 26, 745 (1982).

[16] H. P. Pfeiffer, L. E. Kidder, M. A. Scheel, and D. Shoemaker, Phys. Rev. D 71, 024020 (2005).

[17] H. P. Pfeiffer, L.E. Kidder, M. A. Scheel, and S. A. Teukolsky, Comput. Phys. Commun. 152, 253 (2003).

[18] M. Cantor, Commun. Math. Phys. 57, 83 (1977).

[19] M. Cantor and D. Brill, Compositio Mathematica 43, 317 (1981).

[20] D. Maxwell, Commun. Math. Phys. 253, 561 (2005).

[21] L. Smarr and J. W. York, Jr., Phys. Rev. D 17, 2529 (1978). 